Lígia Cardoso dos Reis ${ }^{1}$

Patricia Constante Jaime ${ }^{2}$

${ }^{1}$ Universidade de São Paulo, Faculdade de Saúde Pública, Programa de Pós-graduação em Nutrição em Saúde Pública. São Paulo, SP, Brasil.

2 Universidade de São Paulo, Faculdade de Saúde Pública, Núcleo de Pesquisas Epidemiológicas em Nutrição e Saúde. São Paulo, SP, Brasil.

Correspondência Lígia Cardoso dos Reis

ligiacreis@hotmail.com

\section{Conhecimento e percepção de autoeficácia e eficácia coletiva de profissionais de saúde para a implementação do Guia Alimentar na Atenção Básica}

Knowledge and perceived self-efficacy and collective efficacy of health professionals to implement the Brazilian Dietary Guidelines in Primary Health Care

\section{Resumo}

Objetivos: Este estudo comparou o conhecimento e a percepção de autoeficácia e eficácia coletiva para aplicar o Guia Alimentar para a População Brasileira [Guia Alimentar] entre nutricionistas e demais profissionais de saúde da Atenção Básica. Métodos: Analisou-se o desempenho de 209 participantes em estudo de validação de constructo de duas escalas: GAB1 (conhecimento) e GAB2 (autoeficácia e eficácia coletiva). Geraram-se grupos segundo percentil (<75 e >75) e testaram-se as diferenças entre variáveis quantitativas/qualitativas e ser nutricionista. Razões de chance por modelos de regressão logística bivariada e múltipla foram calculadas para investigar as variáveis explicativas de cada constructo. Resultados: A média de conhecimento e autoeficácia dos nutricionistas foi estatisticamente superior a das outras profissões, observando-se associação entre ser 
nutricionista e elevado conhecimento e autoeficácia para aplicar o Guia Alimentar. Ser nutricionista e possuir elevada autoeficácia mostraram-se determinantes para elevado conhecimento sobre o Guia Alimentar. Ser nutricionista e possuir elevado conhecimento sobre o Guia Alimentar mostraram-se determinantes para elevada autoeficácia. A média de eficácia coletiva dos nutricionistas não foi estatisticamente diferente a das outras profissões. Nenhuma variável se mostrou explicativa para elevada eficácia coletiva. Discussão: Pelo nosso conhecimento, este estudo é o primeiro a avaliar o preparo de profissionais de saúde para implementar o Guia Alimentar, salientando a urgência de processos eficazes de capacitação profissional para consolidar o nutricionista como referência técnica para os demais profissionais. Conclusões: Ser nutricionista associou-se ao maior conhecimento e à maior autoeficácia para aplicar o Guia Alimentar, mas a eficácia coletiva não esteve associada à profissão.

Palavras-chave: Guias alimentares. Atenção Primária à Saúde. Pessoal de saúde. Autoeficácia. Educação continuada.

\section{Abstract}

Objective: This study compared the knowledge and perceived self-efficacy and collective efficacy to apply the Brazilian Dietary Guidelines (Dietary Guidelines) among nutritionists and other health professionals working in primary health care. Methods: The performance of 209 participants in a construct validation study of two scales was analyzed: GAB1 (knowledge) and GAB2 (self-efficacy and collective efficacy). Groups percentile ( $<75$ and $>75$ ) were generated and the differences between quantitative/qualitative variables and being a nutritionist were tested. Bivariate and multiple logistic regression models were used to investigate the explanatory variables of each construct. Results: Nutritionists had mean knowledge and perceived self-efficacy statistically higher than other professions. We observed association between being a nutritionist and high knowledge and perceived self-efficacy to apply the Dietary Guidelines. Being a nutritionist and having high perceived self-efficacy proved to be determinants of high knowledge about the Dietary Guidelines. Being a nutritionist and having high knowledge about the Dietary Guidelines showed to be determinants of high perceived self-efficacy. Nutritionists' mean collective efficacy was not statistically different than other professions. No variable was found to be predictive of high collective efficacy. Discussion: To our knowledge, this is the first study to evaluate the readiness of health professionals to implement the Dietary Guidelines in Primary Healthcare, showing the urgent need of effective professional training to consolidate the nutritionist as technical reference for other professionals. Conclusions: Being a nutritionist was associated with higher knowledge and perceived self-efficacy to apply the Dietary Guidelines, but collective efficacy was not associated with the profession.

Keywords: Food Guide. Primary Healthcare. Health Personnel. Self-Efficacy. Continuing Education.

\section{INTRODUÇÃO}

A segunda edição do Guia Alimentar para a População Brasileira [Guia Alimentar], publicada em 2014 pelo Ministério da Saúde, ${ }_{1}^{1}$ anunciou um novo paradigma de alimentação saudável, configurando-se como ferramenta de aconselhamento alimentar com recomendações centradas no empoderamento dos sujeitos para escolhas alimentares mais saudáveis, prazerosas, autônomas e conscientes. Esse instrumento incorpora as dimensões sociais, culturais, econômicas e ambientais da sustentabilidade e apresenta recomendações direcionadas à nutrição do corpo, da alma, da mente, da preservação e proteção do planeta e da biosfera. ${ }^{2}$

Implementar as recomendações do Guia Alimentar tornou-se um compromisso do Estado brasileiro com vistas à universalização do acesso à alimentação saudável. ${ }^{3}$ Nesse contexto, a capacitação dos facilitadores da implementação de guias alimentares, entre eles os profissionais de saúde, foi considerada pela Organização das Nações Unidas para Agricultura e Alimentação (FAO) como elemento-chave para subsidiar esse processo. ${ }^{4}$

O Brasil tem incorporado, em diversas políticas públicas e documentos oficiais, a educação permanente como estratégia de qualificação dos profissionais de saúde para a promoção da alimentação adequada e saudável, de acordo com as necessidades de saúde dos usuários do Sistema Único de Saúde (SUS).9-9 Dentre esses documentos, a Política Naciona de Atenção Básica atesta a necessidade de transformação do processo de trabalho das equipes, para que os profissionais de saúde tenham maior capacidade de análise, intervenção e autonomia para o estabelecimento de práticas transformadoras. ${ }^{6}$

Nessa direção, a Teoria Social Cognitiva (TSC), de Albert Bandura, como teoria comportamental bastante empregada no delineamento das intervenções educativas em saúde, 10-12 identifica os diversos constructos influenciadores dos processos de aprendizagem. A TSC 
reconhece o comportamento humano como produto de uma dinâmica articulação de influências pessoais, comportamentais e ambientais. ${ }^{13}$

Dentre esses constructos, o conhecimento é compreendido por Bandura como uma precondição para a mudança em direção à promoção da saúde pelos meios sociais cognitivos. ${ }^{14} \mathrm{~A}$ falta de conhecimento, por sua vez, posiciona os indivíduos numa situação em que poucas razões são encontradas para a mudança comportamental. A TSC destaca que, além do conhecimento, a percepção de autoeficácia é crucial, pois quanto maior a crença de autoeficácia, maiores serão os objetivos que os indivíduos estabelecem para si mesmos e o compromisso assumido com o seu alcance, mesmo na presença de obstáculos. ${ }^{14}$ A TSC considera, ainda, que as pessoas não agem isoladamente. Dessa forma, as crenças que os indivíduos compartilham na eficácia coletiva para realizar uma mudança desempenha papel-chave nas políticas públicas de promoção da saúde e prevenção de doenças. ${ }^{14}$

Para Lindemann et al.,15 os profissionais de saúde são reconhecidamente fundamentais no processo de educação em saúde, cabendo a eles melhor adequar as ações de promoção da alimentação saudável às dificuldades e obstáculos vivenciados pela população. No entanto, desde a publicação do Guia Alimentar, não foram disponibilizados pela literatura panoramas da adequação de sua implementação na Atenção Básica em Saúde (ABS) e sobre o preparo de profissionais de saúde para empregar suas recomendações.

Diante disso, o presente estudo objetivou comparar o conhecimento e a percepção de autoeficácia e eficácia coletiva para aplicar o Guia Alimentar entre nutricionistas e demais profissionais de saúde atuantes na ABS.

\section{METODOLOGIA}

Trata-se de estudo descritivo e transversal para comparar padrões de desempenho entre nutricionistas e outros profissionais de saúde atuantes na ABS em estudo de validação de duas escalas autoaplicáveis orientadas pelo Guia Alimentar. A primeira escala, denominada GAB1, apresenta 16 itens com três opções de respostas ("falso", "verdadeiro" e "não sei") e mensura o conhecimento desses profissionais sobre as recomendações descritas nos cinco capítulos do Guia Alimentar. A segunda escala (GAB2), composta por 24 itens com respostas de quatro pontos na escala Likert, é dividida em duas dimensões para avaliar a percepção de autoeficácia ( 1 =nada confiante até 4=muito confiante) e eficácia coletiva (1=falso até 4=muito verdadeiro) para aplicar esse documento em ações de promoção da alimentação adequada e saudável.

Enquanto GAB1 mensura diretamente o conhecimento do profissional de saúde sobre os conteúdos do Guia Alimentar, GAB2 avalia a percepção de autoeficácia para aplicar esse instrumento, bem como a percepção da atuação da sua equipe de trabalho (eficácia coletiva). Dessa forma, os conteúdos abordados nos itens da dimensão autoeficácia da GAB2 correspondem aos conteúdos dos itens da dimensão eficácia coletiva, conforme exemplos citados no quadro 1.

Quadro 1. Exemplos de itens da GAB1 e GAB2, 2017.

\begin{tabular}{|c|c|c|}
\hline Escala & Exemplo de item & Opções de resposta \\
\hline GAB1 & \multirow{3}{*}{$\begin{array}{l}\text { Uma alimentação em que } \\
\text { predominam os alimentos } \\
\text { ultraprocessados é mais barata } \\
\text { que aquela em que predominam } \\
\text { os alimentos in natura ou } \\
\text { minimamente processados }\end{array}$} & 1- Verdadeiro \\
\hline \multirow{2}{*}{$\begin{array}{l}\text { (Escala de avaliação do } \\
\text { conhecimento de profissionais de } \\
\text { saúde da Atenção Básica sobre os } \\
\text { conteúdos do Guia Alimentar) }\end{array}$} & & 2- Falso \\
\hline & & 3- Não sei \\
\hline \multirow{4}{*}{$\begin{array}{l}\text { GAB2 } \\
\text { (Escala de avaliação da percepção } \\
\text { de autoeficácia e eficácia coletiva } \\
\text { de profissionais de saúde da } \\
\text { Atenção Básica para aplicar o Guia } \\
\text { Alimentar: dimensão autoeficácia) }\end{array}$} & \multirow{4}{*}{$\begin{array}{l}\text { Eu consigo orientar os usuários } \\
\text { do serviço de saúde sobre como } \\
\text { economizar com a compra de } \\
\text { alimentos saudáveis. }\end{array}$} & 1- nada confiante \\
\hline & & 2- um pouco confiante \\
\hline & & 3- confiante \\
\hline & & 4- muito confiante \\
\hline \multirow{4}{*}{$\begin{array}{l}\text { GAB2 } \\
\text { (Escala de avaliação da percepção } \\
\text { de autoeficácia e eficácia coletiva } \\
\text { de profissionais de saúde da } \\
\text { Atenção Básica para aplicar o } \\
\text { Guia Alimentar: dimensão eficácia } \\
\text { coletiva) }\end{array}$} & \multirow{4}{*}{$\begin{array}{l}\text { A minha equipe é capaz de orientar } \\
\text { os usuários do serviço de saúde } \\
\text { sobre como economizar com a } \\
\text { compra de alimentos saudáveis. }\end{array}$} & 1 - falso \\
\hline & & 2- às vezes verdadeiro \\
\hline & & 3-verdadeiro \\
\hline & & 4- muito verdadeiro \\
\hline
\end{tabular}

GAB1 e GAB2 passaram por processos de validação de conteúdo, aparente e de constructo, por meio de análise fatorial. A presente pesquisa foi realizada com a amostra de participantes do estudo de validação de constructo, para comparar os padrões de resposta entre nutricionistas e demais profissionais atuantes na ABS. Decidiu-se trabalhar com esses dados, tendo em vista que as escalas foram consideradas precisas para avaliar profissionais com baixo conhecimento (abaixo da média) sobre o Guia Alimentar e percepção de autoeficácia e eficácia coletiva na média.

Em relação ao constructo mensurado pela GAB1, o conhecimento na Psicologia é compreendido como condição para explicarmos aspectos importantes do mundo e prevermos eventos, podendo ser agrupado em declarativo (saber o que são coisas e processos) e procedural (saber como fazer coisas e processos). ${ }^{16}$ 
Quanto aos constructos mensurados pela GAB2, a autoeficácia é definida como a confiança ou crença na habilidade que uma pessoa possui para adotar determinado comportamento, enquanto a eficácia coletiva compreende a confiança ou crença na habilidade de um grupo agir em direção ao alcance das mudanças almejadas e a disposição de membros de uma comunidade intervirem para apoiar uns aos outros. ${ }^{13}$

A população de estudo foi convidada, em redes sociais das pesquisadoras e pelo site da Faculdade de Saúde Pública/USP, para acessar a plataforma online elaborada para a coleta de dados entre abril e junho de 2017. Para evitar duplicidade de respostas, a plataforma fo programada para registrar o endereço IP do computador do respondente. Foram considerados elegíveis os profissionais de saúde atuantes na ABS por pelo menos um ano. A plataforma incluiu seção com informações pessoais e sobre a atuação profissional do respondente, para então dar início ao registro de respostas da GAB1 e da GAB2.

Realizou-se análise descritiva das características dos respondentes (nutricionistas e outras profissões), sendo as variáveis qualitativas apresentadas em seus valores absolutos e relativos, e as variáveis quantitativas, em seus valores de tendência central e dispersão. Foram excluídas das análises todas as respostas em branco referentes à caracterização dos respondentes. Para avaliar a normalidade da distribuição dos dados, utilizou-se o teste de Shapiro-Wilk.

Calculou-se a soma final dos acertos de cada respondente para a GAB1 (conhecimento sobre o Guia Alimentar), considerando a resposta "não sei" como incorreta, a fim de classificá-los em dois grupos: "elevado conhecimento" (percentil 75 ou mais) e "baixo conhecimento" (percentil menor que 75).

Os resultados da aplicação da GAB2 foram agrupados de acordo com cada dimensão: o desempenho nos 12 itens de autoeficácia gerou os grupos "não confiantes" (respostas registradas como "nada confiante" e "um pouco confiante") e "confiantes" (respostas registradas como "confiante" e "muito confiante"), e os 12 itens de eficácia coletiva geraram os grupos "falso" (respostas registradas como "falso" e "às vezes verdadeiro") e "verdadeiro" (respostas registradas como "verdadeiro" e "muito verdadeiro"). Foram calculados os percentis da soma de respostas "confiantes" e "verdadeiro", para classificá-las em dois grupos: percentis 75 ou mais (respondentes com "elevada autoeficácia" e "elevada eficácia coletiva") e percentis menores que 75 (respondentes com "baixa autoeficácia" e "baixa eficácia coletiva").

Foram testadas as diferenças entre as variáveis quantitativas e "ser nutricionista" com o teste de Mann-Whitney, e avaliada a associação entre as variáveis qualitativas e "ser nutricionista" com o teste exato de Fisher.

Como medida de associação entre elevado conhecimento sobre o Guia Alimentar (percentil 75 ou mais) e as possíveis variáveis explicativas (ser nutricionista, faixa etária, sexo, tempo de atuação na ABS, atuação no Núcleo de Apoio à Saúde da Família - NASF, formação superior, percepção de autoeficácia e percepção de eficácia coletiva), foram calculadas as razões de chance (OR) por modelos de regressão logística bivariada e múltipla. Nos modelos múltiplos, foram incluídas as variáveis que tiveram valor de $p$ menor que 0,20. O mesmo procedimento foi seguido para avaliar a associação entre a elevada percepção de autoeficácia ("elevada autoeficácia") e de eficácia coletiva ("elevada eficácia coletiva") com as possíveis variáveis explicativas.

Todas as análises estatísticas foram feitas no software STATA versão 13.1, sendo adotado nível de significância menor ou igual a 0,05.

Esta pesquisa foi aprovada pelo Comitê de Ética em Pesquisa da Faculdade de Saúde Pública da USP - protocolo número 56303716.6.0000.5421. A participação foi voluntária e todos os participantes forneceram consentimento informado.

\section{RESULTADOS}

GAB1 e GAB2 foram submetidas à validação de constructo com 209 profissionais de saúde atuantes na ABS, sendo 133 nutricionistas (63,6\%). As demais profissões incluíram 32 enfermeiros (15,3\%), oito médicos (3,8\%), oito agentes comunitários de saúde (3,8\%), cinco assistentes sociais (2,4\%), cinco fonoaudiólogos (2,4\%), cinco psicólogos (2,4\%), cinco profissionais de educação física (2,4\%) e oito profissionais de áreas diversas (3,8\%). Todas as macrorregiões brasileiras foram representadas neste estudo: $52,2 \%$ dos profissionais de saúde atuavam no Sudeste; 20,1\% no Sul; 17,2\% no Nordeste; 6,7\% no Centro-Oeste; e 3.8\% no Norte.

A tabela 1 apresenta as variáveis descritivas dos respondentes de acordo com a profissão. A média de idade foi de 38 anos (desvio padrão - DP =9,05), sendo que os nutricionistas apresentaram idade média (37 anos, DP=9,12) estatisticamente diferente $(p=0,002)$ dos demais profissionais de saúde (40 anos, $D P=8,55)$. Além da idade, os dois grupos apresentaram diferenças em relação à distribuição por sexo, tempo de atuação na ABS, atuação no NASF e formação superior.

A média de acertos dos nutricionistas na GAB1 foi estatisticamente superior $(14,18$, $\mathrm{DP}=1,71)$ a das outras profissões $(11,52, \mathrm{DP}=2,47)(\mathrm{p}=0,000)$. A tabela 2 demonstra a associação existente entre ser nutricionista e apresentar elevado conhecimento sobre o Guia Alimentar.

Os resultados descritivos do desempenho na GAB2 demonstraram que a média de percepção da autoeficácia dos nutricionistas foi de 9,42 (DP=1,98), enquanto entre as outras profissões foi de 6,31 ( $\mathrm{DP}=2,94)$, sendo estatisticamente diferentes $(\mathrm{p}=0,000)$. A tabela 2 demonstra a associação existente entre ser nutricionista e apresentar elevada percepção de autoeficácia para aplicar o Guia Alimentar. 
Tabela 1. Características Sociodemográficas pela Profissão do Respondente, 2017.

\begin{tabular}{|c|c|c|}
\hline \multirow{2}{*}{ Variável } & Nutricionistas & Outras Profissões \\
\hline & \multicolumn{2}{|c|}{$\mathrm{N}(\%)$} \\
\hline \multicolumn{3}{|l|}{ Faixa etária (N=208)a } \\
\hline$<25$ anos & $4(3,03)$ & $0(0,00)$ \\
\hline 25 a 34 anos & $56(42,42) \mathrm{b}$ & $19(25,00)$ \\
\hline 35 a 44 anos & $46(34,85)$ & $33(43,42)$ \\
\hline 45 a 54 anos & $17(12,88)$ & $20(26,32)$ \\
\hline 55 anos ou mais & $9(6,82)$ & $4(5,26)$ \\
\hline Total & $132(100,00)$ & $76(100,00)$ \\
\hline \multicolumn{3}{|l|}{$\operatorname{Sexo}(N=209)$} \\
\hline Feminino & $128(96,24)^{b}$ & $63(82,89)$ \\
\hline Masculino & $5(3,76)$ & $13(17,11)$ \\
\hline Total & $133(100,00)$ & $76(100,00)$ \\
\hline \multicolumn{3}{|c|}{ Tempo de atuação na ABS ( $N=209$ ) } \\
\hline$<5$ anos & $54(40,60)$ & $18(23,68)$ \\
\hline 5 anos ou mais & $79(59,40) \mathrm{b}$ & $58(76,32)$ \\
\hline Total & $133(100,00)$ & $76(100,00)$ \\
\hline \multicolumn{3}{|c|}{ Atuação no NASF (N=209) } \\
\hline Sim & $58(43,61)$ & $20(26,32)$ \\
\hline Não & $75(56,39) \mathrm{b}$ & $56(73,68)$ \\
\hline Total & $133(100,00)$ & $76(100,00)$ \\
\hline \multicolumn{3}{|c|}{ Formação superior ( $N=209)$} \\
\hline Sim & $133(100,00) \mathrm{b}$ & $67(88,16)$ \\
\hline Não & $0(0,00)$ & $9(11,84)$ \\
\hline Total & $133(100,00)$ & $76(100,00)$ \\
\hline \multicolumn{3}{|c|}{ Tempo de graduação $(N=200)^{a}$} \\
\hline Até 10 anos & $65(48,87)$ & $27(40,30)$ \\
\hline$>10$ anos & $68(51,13)$ & $40(59,70)$ \\
\hline Total & $133(100,00)$ & $67(100,00)$ \\
\hline \multicolumn{3}{|c|}{ Pós-graduação (N=200)a } \\
\hline Sim & $114(85,71)$ & $51(76,12)$ \\
\hline Não & $19(14,29)$ & $16(23,88)$ \\
\hline Total & $133(100,00)$ & $67(100,00)$ \\
\hline
\end{tabular}

Tabela 1. Características Sociodemográficas pela Profissão do Respondente, 2017. (cont.)

\begin{tabular}{lcc}
\hline Variável & Nutricionistas & Outras Profissões $^{c}$ \\
\cline { 2 - 3 } Macrorregiões de atuação (N=209) & & \\
Norte & $7(5,26)$ & $2(2,63)$ \\
Nordeste & $22(16,54)$ & $13(17,11)$ \\
Centro-Oeste & $13(9,77)$ & $2(2,63)$ \\
Sudeste & $65(48,87)$ & $44(57,89)$ \\
Sul & $26(19,55)$ & $15(19,74)$ \\
Total & $133(100,00)$ & $76(100,00)$ \\
\hline
\end{tabular}

a Missing = respostas não fornecidas

'Teste exato de Fisher $p<0,05$

c Outras profissões: enfermeiros, médicos, agentes comunitários de saúde, assistentes sociais, fonoaudiólogos, psicólogos, profissionais de educação física e outros profissionais de áreas diversas.

ABS = Atenção Básica em Saúde

NASF = Núcleo de Apoio à Saúde da Família

Tabela 2. Conhecimento e percepção de autoeficácia e eficácia coletiva dos respondentes segundo profissão, 2017.

\begin{tabular}{lccc}
\hline & Nutricionistas & Outras Profissões & Total \\
\hline Conhecimento - GAB1 (categoria) & & $\mathrm{N}(\%)$ & \\
$1^{\circ}$ ao $3^{\circ}$ quarto & $66(49,62)$ & $66(86,84)$ & $132(63,16)$ \\
$4^{\circ}$ quarto & $67(50,38) a$ & $10(13,16)$ & $77(36,84)$ \\
Autoeficácia - GAB2 (categoria) & & $\mathrm{N}(\%)$ & \\
$1^{\circ}$ ao $3^{\circ}$ quarto & $76(51,14)$ a & $70(92,11)$ & $146(69,86)$ \\
$4^{\circ}$ quarto & $57(42,86)$ & $6(7,89)$ & $63(30,14)$ \\
Eficácia coletiva - GAB2 (categoria) & & $\mathrm{N}(\%)$ & \\
$1^{\circ}$ ao $3^{\circ}$ quarto & $94(70,68)$ & $53(69,74)$ & $147(70,33)$ \\
$4^{\circ}$ quarto & $39(29,32)$ & $23(30,26)$ & $62(29,67)$ \\
\hline
\end{tabular}


Por fim, a média obtida para percepção de eficácia coletiva (GAB2) dos nutricionistas fo de 4,93 ( $D P=3,94)$, enquanto entre as outras profissões foi de 5,46 ( $D P=3,54)$, não sendo estatisticamente diferentes $(p=0,189)$. A tabela 2 demonstra que não foi identificada associação estatisticamente significativa entre a profissão e a percepção de eficácia coletiva para aplicar o Guia Alimentar.

Entre as possíveis variáveis explicativas para possuir elevado conhecimento do Guia Alimentar (percentil 75 ou mais de acertos na GAB1), estão ser nutricionista e possuir elevada percepção de autoeficácia (percentil 75 ou mais na GAB2 - autoeficácia), segundo a tabela 3.

No modelo múltiplo, ser nutricionista foi importante fator para possuir elevado conhecimento sobre o Guia Alimentar, com uma chance cinco vezes maior de estar acima do percentil 75 de acertos na GAB1, se comparado aos demais profissionais da ABS, ajustado pela formação superior e pela elevada percepção de autoeficácia na GAB2 (tabela 3).

As possíveis variáveis explicativas para possuir elevada percepção de autoeficácia (acima do percentil 75 na GAB2) são "ser nutricionista" (tabela 4) e "apresentar elevado conhecimento sobre o Guia Alimentar" (acima do percentil 75 de acertos na GAB1), ainda segundo a tabela 3.

No modelo múltiplo, ser nutricionista se mostrou como importante determinante para a elevada percepção de autoeficácia, com uma chance sete vezes maior se comparado aos demais profissionais da ABS, ajustado por "atuar no NASF", "possuir elevado conhecimento sobre o Guia Alimentar" (percentil 75 ou mais de acertos na GAB1) e pela "elevada percepção eficácia coletiva" (percentil 75 ou mais de acertos na GAB2) (tabela 4).

Entre as possíveis variáveis explicativas para possuir elevada percepção de eficácia coletiva (percentil 75 ou mais na GAB2), nenhuma se mostrou estatisticamente significativa (tabela 4). Assim como no modelo bivariado, no modelo múltiplo nenhuma variável explicativa se mostrou como importante determinante para a eficácia coletiva (tabela 4).

\section{DISCUSSÃO}

O presente estudo se propôs a comparar o preparo de nutricionistas e demais profissionais de saúde (não nutricionistas) atuantes na ABS para empregar o Guia Alimentar nas ações de promoção da alimentação adequada e saudável. Esse preparo foi avaliado por meio da análise do conhecimento desses dois grupos sobre as recomendações da referida publicação do Ministério da Saúde, ${ }^{1}$ bem como da percepção de autoeficácia e eficácia coletiva para utilizá-las em suas aç̃os educativas. Os resultados descritos neste estudo integram etapa de validação de constructo de duas escalas (GAB1 e GAB2) orientadas pelo Guia Alimentar.
Tabela 3. Odds ratio bruto (OR) e odds ratio ajustado (ORa) com os respectivos intervalos de confiança e valor de $p$ para percentil 75 ou mais obtidos na GAB1 (conhecimento) segundo variáveis de interesse, 2017.

\begin{tabular}{|c|c|c|}
\hline & OR [IC95\%] & $P$ \\
\hline Nutricionista (Referência: não ser nutricionista) & 1 & \\
\hline Ser nutricionista & $6,70[3,17-14,14]$ & 0,000 \\
\hline Faixa etária (Referência: menos de 25 anos) & 1 & \\
\hline 25 a 34 anos & $1,89[0,18-19,06]$ & 0,589 \\
\hline 35 a 44 anos & $1,83[0,18-18,47]$ & 0,606 \\
\hline 45 a 54 anos & $1,11[0,10-11,96]$ & 0,931 \\
\hline 55 anos ou mais & $2,57[0,20-31,70]$ & 0,461 \\
\hline Sexo (Referência: feminino) & 1 & \\
\hline Masculino & $1,10[0,40-2,96]$ & 0,851 \\
\hline Tempo atuação na Atenção Básica (Referência: < 5 anos) & 1 & \\
\hline 5 anos ou mais & $0,95[0,53-1,72]$ & 0,886 \\
\hline Atuar no NASF (Referência: não) & 1 & \\
\hline Sim & $1,02[0,57-1,82]$ & 0,938 \\
\hline Formação superior (Referência: não) & 1 & \\
\hline $\operatorname{sim}$ & $4,90[0,60-39,97]$ & 0,138 \\
\hline Percepção de autoeficácia - GAB2 (Referência: $1^{\circ}$ a $3^{\circ}$ quarto) & 1 & \\
\hline A partir do $4^{\circ}$ quarto & $3,09[1,67-5,71]$ & 0,000 \\
\hline Percepção de eficácia coletiva - GAB2 (Referência: $1^{\circ}$ a $3^{\circ}$ quarto) & 1 & \\
\hline \multirow[t]{2}{*}{ A partir do $4^{\circ}$ quarto } & $0,92[0,49-1,70]$ & 0,792 \\
\hline & ORa [IC95\%] & $P$ \\
\hline Nutricionista (Referência: não ser nutricionista) & 1 & \\
\hline Ser nutricionista & $5,30[2,35-11,93]$ & 0,000 \\
\hline Formação superior (Referência: não) & 1 & \\
\hline Sim & $1,27[0,14-11,93]$ & 0,827 \\
\hline Percepção de autoeficácia - GAB2 (Referência: $1^{\circ}$ a $3^{\circ}$ quarto) & 1 & \\
\hline A partir do $4^{\circ}$ quarto & $1,86[0,96-3,61]$ & 0,064 \\
\hline
\end{tabular}

NASF = Núcleo de Apoio à Saúde da Família 
Tabela 4. Odds ratio bruto (OR) e odds ratio ajustado (ORa) com os respectivos intervalos

de confiança e valor de $p$ para percentil 75 ou mais obtidos na GAB2 (autoeficácia e eficácia coletiva) segundo variáveis de interesse, 2017.

\begin{tabular}{|c|c|c|}
\hline GAB2 - Percepção de autoeficácia & OR [IC95\%] & $P$ \\
\hline Nutricionista (Referência: não ser nutricionista) & 1 & \\
\hline Ser nutricionista & $8,75[3,55-21,55]$ & 0,000 \\
\hline Faixa etária (Referência: menos de 25 anos) & 1 & \\
\hline 25 a 34 anos & $1,24[0,12-12,63]$ & 0,853 \\
\hline 35 a 44 anos & $1,15[0,11-11,73]$ & 0,901 \\
\hline 45 a 54 anos & $1,11[0,10-11,96]$ & 0,931 \\
\hline 55 anos ou mais & $4,80[0,38-59,89]$ & 0,223 \\
\hline Sexo (Referência: feminino) & 1 & \\
\hline Masculino & $0,63[0,20-2,02]$ & 0,447 \\
\hline Tempo atuação na Atenção Básica (Referência: < 5 anos) & 1 & \\
\hline 5 anos ou mais & $1,18[0,63-2,23]$ & 0,589 \\
\hline Atuar no NASF (Referência: não) & 1 & \\
\hline Sim & $1,68[0,92-3,08]$ & 0,089 \\
\hline Formação superior (Referência: não) & 1 & \\
\hline Sim & $3,59[0,43-29,36]$ & 0,233 \\
\hline Percepção de eficácia coletiva - GAB2 (Referência: $1^{\circ}$ a $3^{\circ}$ quarto) & 1 & \\
\hline A partir do $4^{\circ}$ quarto & $1,74[0,93-3,27]$ & 0,081 \\
\hline GAB2 - Percepção de Autoeficácia & ORa [IC95\%] & $P$ \\
\hline Nutricionista (Referência: não ser nutricionista) & 1 & \\
\hline Ser nutricionista & $7,02[2,72-18,07]$ & 0,000 \\
\hline Atuar no NASF (Referência: não) & 1 & \\
\hline Sim & $1,33[0,68-2,58]$ & 0,398 \\
\hline Conhecimento - GAB1 (Referência: $1^{\circ} \mathrm{a} 3^{\circ}$ quarto) & 1 & \\
\hline A partir do $4^{\circ}$ quarto & $1,98[1,01-3,89]$ & 0,045 \\
\hline Percepção de eficácia coletiva - GAB2 (Referência: $1^{\circ}$ a $3^{\circ}$ quarto) & 1 & \\
\hline A partir do $4^{\circ}$ quarto & $2,00[0,99-4,03]$ & 0,053 \\
\hline GAB2 - Percepção de Eficácia coletiva & OR [IC95\%] & $P$ \\
\hline Nutricionista (Referência: não ser nutricionista) & 1 & \\
\hline Ser nutricionista & $0,95[0,51-1,76]$ & 0,886 \\
\hline
\end{tabular}

Tabela 4. Odds ratio bruto (OR) e odds ratio ajustado (ORa) com os respectivos intervalos de confiança e valor de $p$ para percentil 75 ou mais obtidos na GAB2 (autoeficácia e eficácia coletiva) segundo variáveis de interesse, 2017. (cont.)

\begin{tabular}{|c|c|c|}
\hline GAB2 - Percepção de Eficácia coletiva & OR [IC95\%] & $P$ \\
\hline Faixa etária (Referência: menos de 25 anos) & 1 & \\
\hline 25 a 34 anos & $1,16[0,11-11,85]$ & 0,896 \\
\hline 35 a 44 anos & $1,64[0,16-16,58]$ & 0,672 \\
\hline 45 a 54 anos & $0,82[0,07-9,07]$ & 0,877 \\
\hline 55 anos ou mais & $1,33[0,10-17,09]$ & 0,825 \\
\hline Sexo (Referência: feminino) & 1 & \\
\hline Masculino & $0,90[0,30-2,65]$ & 0,855 \\
\hline Tempo atuação na Atenção Básica (Referência: <5 anos) & 1 & \\
\hline 5 anos ou mais & $0,76[0,41-1,42]$ & 0,401 \\
\hline Atuar no NASF (Referência: não) & 1 & \\
\hline Sim & $1,75[0,95-3,21]$ & 0,068 \\
\hline Formação superior (Referência: não) & 1 & \\
\hline Sim & $0,83[0,20-3,45]$ & 0,806 \\
\hline GAB2 - Percepção de eficácia coletiva & ORa [IC95\%] & $P$ \\
\hline Atuar no NASF (Referência: não) & 1 & \\
\hline Sim & $1,66[0,90-3,07]$ & 0,101 \\
\hline Percepção de autoeficácia - GAB2 (Referência: $1^{\circ}$ a $3^{\circ}$ quarto) & 1 & \\
\hline A partir do $4^{\circ}$ quarto & $1,65[0,87-3,11]$ & 0,122 \\
\hline
\end{tabular}

NASF = Núcleo de Apoio à Saúde da Família

A análise global do desempenho dos respondentes da GAB1 concluiu que ser nutricionista e possuir elevada percepção de autoeficácia mostraram-se como variáveis explicativas para o elevado conhecimento sobre o Guia Alimentar, com uma chance cinco vezes maior de nutricionistas estarem acima do percentil 75 de acertos, se comparados aos demais profissionais da ABS. Da mesma forma, ser nutricionista e possuir elevado conhecimento sobre o Guia Alimentar mostraram-se como variáveis explicativas para a elevada percepção de autoeficácia, com uma chance sete vezes maior de nutricionistas estarem acima do percentil 75 de acertos para autoeficácia na GAB2, comparados aos demais profissionais da ABS. 
Os resultados deste estudo sugerem que os nutricionistas foram os profissionais que mais acessaram as recomendações e diretrizes dessa publicação, seja de forma autônoma ou com processos formativos destinados à qualificação das ações de promoção da alimentação adequada e saudável.

Embora o Guia Alimentar tenha sido publicado em 2014 como um instrumento de apoio às ações de educação em saúde para todos os profissionais de saúde, é frequente no país o entendimento de que os cuidados relativos a alimentação e nutrição sejam de responsabilidade exclusiva do nutricionista, e não sejam compartilhados entre os demais membros da equipe de saúde. ${ }^{17,18}$ Os achados do presente estudo podem refletir, portanto, o quanto as ações de educação alimentar e nutricional (EAN) foram estabelecidas historicamente no país como campo de prática exclusivo do nutricionista. Essa concepção tem disseminado intervenções fragmentadas que não atendem às demandas da população e do sistema de saúde. ${ }^{17}$ Para Cervato-Mancuso et al..19 ${ }^{19}$ Marco de Referência de Educação Alimentar e Nutricional para as Políticas Públicas trouxe a reorientação das práticas de EAN e salientou a importância de profissionais não nutricionistas planejarem e executarem essas ações. Transcorridos seis anos da publicação do Marco de EAN, o presente estudo sugere que as recomendações do Guia Alimentar podem ter sido mais acessadas e valorizadas pelos nutricionistas.

Para a FAO, ${ }^{4}$ a maioria dos países contam com planos de implementação de guias alimentares que desconsideram estratégias integrais de comunicação, sendo que, na existência dessas, faltam apoio político e recursos para executá-las. Não foram encontrados estudos que tenham avaliado o preparo de profissionais de saúde para implementar o Guia Alimentar em ações de EAN no país ou que tenham analisado o processo de implementação desse documento na ABS. Pelo nosso conhecimento, este estudo é o primeiro a realizar tal diagnóstico.

Estudo de Ashman et al..$^{20}$ observou que a autoeficácia de clínicos gerais australianos para o tratamento da obesidade foi afetada pelas práticas e experiências prévias desses profissionais, bem como por suas percepções de conhecimentos e habilidades. A base de conhecimento e as habilidades desenvolvidas na vida profissional foram reconhecidas como os fatores sobre os quais os profissionais exercem maior controle para modificar os contextos vivenciados. A TSC de Bandura posiciona o conhecimento como uma premissa importante para a maior autoeficácia em direção ao alcance de metas traçadas pelos sujeitos. ${ }^{14} \mathrm{Com}$ maior bagagem de conhecimento, as pessoas encontram razões para seguirem em direção aos objetivos almejados. Estes, por sua vez, serão mais robustos à medida que o indivíduo fortalece sua crença de autoeficácia e encontra meios para lidar e superar os obstáculos encontrados pelo caminho.

Sendo assim, os achados deste estudo salientam a urgência do planejamento e da implementação de processos mais amplos e eficazes de educação permanente direciona- dos à aplicação do Guia Alimentar com todos os profissionais de saúde responsáveis pela promoção da alimentação saudável e pela organização da atenção nutricional no SUS, tendo a ABS como ordenadora da Rede de Atenção à Saúde (RAS). As RAS configuram-se como um sistema que busca aprofundar e estabelecer inter-relações, ampliando a capacidade de aprendizagem, a difusão de conhecimentos e a utilização das informações existentes para a geração de novos conhecimentos..$^{21}$ Dessa forma, investigar como os profissionais de saúde atuantes nessas redes estão implementando as recomendações do Guia Alimentar torna-se fundamental como diagnóstico para políticas públicas de educação permanente em alimentação saudável.

Silva et al. problematizaram em seu estudo o paradigma de alimentação saudável assumido pelos profissionais de saúde atuantes na ABS.22 Essa publicação ressaltou a necessidade de superação do paradigma do discurso biomédico, centrado na doença e nos nutrientes, para atuar com o indivíduo de forma plena. Os autores apresentaram como desafio do milênio a exigência de profissionais de saúde capazes de associar saberes e práticas potencializadores do seu papel de agentes de promoção da saúde coletiva, viabilizando o resgate da totalidade do ser humano. Nessa direção, entre os compromissos assumidos pelo Brasil para a Década de Ação das Nações Unidas para a Nutrição (2016-2025), ${ }^{3}$ destacou-se a promoção do acesso universal à alimentação adequada e saudável, com prioridade para as famílias e pessoas em situação de insegurança alimentar e nutricional, povos e comunidades tradicionais e outros grupos socialmente vulneráveis. A implementação das recomendações do Guia Alimentar é citada como estratégica para esse compromisso ser firmado, por estimular o consumo de alimentos regionais e as práticas produtivas sustentáveis que respeitem a biodiversidade. ${ }^{3}$

Embora nenhuma variável explicativa tenha se mostrado determinante para a eficácia coletiva dos profissionais de saúde para a aplicação do Guia Alimentar, esperava-se constatar a atuação no NASF como um importante determinante da elevada percepção de eficácia coletiva. Nutricionistas atuantes no NASF tendem a assumir modelo direcionado à atuação multidisciplinar, focando no atendimento em grupo para a promoção da saúde e tratando o indivíduo em sua integridade. ${ }^{17-19}$

Vale ressaltar que, neste estudo, a elevada percepção de autoeficácia de nutricionistas para aplicar o Guia Alimentar não afetou a eficácia coletiva dos demais profissionais, comprovando que a crença na eficácia coletiva não resulta simplesmente da soma das percepções individuais de eficácia, mas como uma propriedade do grupo. Para Bandura, ${ }^{23}$ as realizações conquistadas por um grupo acabam sendo produto não apenas das habilidades e do conhecimento que seus membros compartilham, mas também de suas ações interativas, coordenadas e sinérgicas. Essas interações fazem com que as pessoas alcancem muitos dos seus objetivos apenas com esforços interdependentes. 
Campos, em 2000, já anunciava o relevante enfraquecimento da Saúde Pública como especialidade, e o descontrole sobre a qualidade dos profissionais autorizados a exercer as atividades de Saúde Coletiva. ${ }^{24}$ Esse autor salientou a importância da combinação de duas perspectivas para contornar esse cenário: socializar saberes e práticas e assegurar especialistas aptos à produção de saberes mais sofisticados em Saúde Pública, para intervirem em situações complexas. Para Campos, ${ }^{24}$ todas as profissões de saúde deveriam abarcar, em sua formação e em sua prática, elementos da Saúde Coletiva para que a missão desse campo fosse conquistada: transformar saberes e práticas para modificar o modelo de atenção e a lógica de funcionamento dos serviços de saúde.

Este estudo sugere, portanto, que a lógica da atuação em equipe, interdisciplinar e do apoio matricial, pode não estar sendo atendida na ABS para a efetivação das ações de EAN calcadas no Guia Alimentar. Pesquisadores têm chamado a atenção para as falhas no preparo de profissionais de saúde para a atuação colaborativa interprofissional a fim de atender às crescentes e cada vez mais complexas demandas de saúde das populações. ${ }^{25-27}$

A TSC considera que as crenças que os indivíduos compartilham na eficácia coletiva para realizar uma mudança desempenha papel-chave nas políticas públicas de promoção da saúde e prevenção de doenças, uma vez que as pessoas não agem isoladamente. ${ }^{14}$ Bandura descreve que muitos dos desafios e dificuldades individuais refletem problemas vivenciados por um grupo, requerendo apoio coletivo para serem superados, uma vez que as pessoas não vivem socialmente isoladas. ${ }^{28}$ Isso reforça o quanto o senso de autoeficácia não está desconectado da percepção de eficácia coletiva e o quanto a eficácia coletiva está impregnada na autoeficácia. Afinal, pessoas com boa percepção de eficácia coletiva mobilizam esforços e recursos para superar obstáculos do ambiente externo e conquistar aquilo que desejam. Por outro lado, aqueles convencidos da sua ineficácia desistem de tentar, mesmo quando mudanças sejam viáveis com o esforço coletivo.

Nesse contexto de qualificação dos profissionais, o nutricionista deve atuar como matriciador das equipes da Estratégia Saúde da Família, com vistas a potencializar ações de alimentação e nutrição, auxiliando os demais profissionais de saúde das equipes a superarem as dificuldades que enfrentam com a implementação do Guia Alimentar. O nutricionista contribui para a definição de uma agenda integrada de organização da atenção nutricional, a partir das necessidades das equipes e das comunidades dos territórios de atuação. ${ }^{18}$ Neste estudo, ficou evidente a incapacidade do NASF de superar a lógica fragmentada da saúde e desconectada das diretrizes de ação interdisciplinar e intersetorial, necessárias para consolidar ações efetivas de promoção da alimentação adequada e saudável e assegurar qualidade da atenção nutricional na ABS. Esse entrave parece ser contornável com ações de educação permanente que considerem a lógica do matriciamento e da interdisciplinaridade na ABS para a efetiva implementação das diretrizes, recomendações e paradigma de alimentação saudável preconizados pelo Guia Alimentar.

A educação permanente em saúde promove a autovalorização profissional e a valorização proveniente de outros profissionais de saúde e da população, minimizando os efeitos do modelo biomédico. ${ }^{29} \mathrm{O}$ predomínio desse modelo curativista e hospitalocêntrico contribui para posicionar em segundo plano as estratégias de promoção da alimentação adequada e saudável no SUS. ${ }^{30}$ Como consequência, dificuldades em adotar padrões alimentares saudáveis já foram reportadas entre usuários da ABS, demonstrando a necessidade de implementação de ações mais efetivas nesse contexto. ${ }^{15}$

Dessa forma, o presente estudo salienta a urgência de processos mais eficazes de capacitação de profissionais de saúde atuantes na ABS para a implementação do Guia Alimentar, de forma a viabilizar a consolidação do nutricionista como referência técnica para os demais membros das equipes de saúde também planejarem e executarem ações de promoção da alimentação adequada e saudável.

Embora este estudo tenha sido realizado com profissionais de saúde atuantes em todas as macrorregiões do país, vale ressaltar que foi utilizada uma amostra de conveniência, o que impossibilita extrapolar os resultados para o universo de profissionais de saúde da ABS no Brasil. Ainda assim, seus achados descrevem de forma inédita o preparo dessa população para implementar o Guia Alimentar, publicado em 2014 pelo Ministério da Saúde.

\section{CONCLUSÕES}

O presente estudo concluiu que ser nutricionista associou-se não apenas a possuir maior conhecimento das recomendações do Guia Alimentar, como também maior autoeficácia para aplicá-las. No entanto, a eficácia coletiva para empregar o Guia Alimentar não esteve associada à profissão.

\section{FINANCIAMENTO}

Esta pesquisa foi financiada pelo International Development Research Centre (IDRC) processo n CR-23447, e pelo Conselho Nacional de Desenvolvimento Científico e Tecnológico (CNPq), processo n 408115/2017-3. As agências financiadoras não estiveram envolvidas no delineamento do estudo, na coleta, análise e interpretação dos dados ou redação do manuscrito. 


\section{REFERÊNCIAS}

1. Ministério da Saúde. Secretaria de Atenção à Saúde. Departamento de Atenção Básica. Guia Alimentar para a População Brasileira. Brasília: 2014. 152 f. [acesso em 29 março 2019]. Disponível em: http://bvsms.saude.gov.br/bvs/publicacoes/guia_alimentar_populacao_brasileira_2ed.pdf

2. Monteiro CA, Cannon G, Moubarac J, Martins APB, Martins CA, Garzillo J, et al. Dietary guidelines to nourish humanity and the planet in the twenty-first century. A blueprint from Brazil. Public Health Nutr 2015;18(13): 2311-22.

3. Câmara Interministerial de Segurança Alimentar e Nutricional (CAISAN). Compromissos do Brasil para a Década de Ação das Naç̃̃es Unidas para a Nutrição (2016-2025). Brasília: CAISAN; 2015. 18 p.

4. Organización de las Naciones Unidas para la Alimentación y la Agricultura (FAO). El estado de las guías alimentarias baseadas en alimentos en América Latina y el Caribe: 21 años después de la Conferencia Internacional sobre Nutrición. Roma: 2014. 125 f. [acesso em 29 março 2019]. Disponível em: http://www.fao.org/3/a-i3677s.pdf

5. Ministério da Saúde. Plano de Ações Estratégicas para o Enfrentamento das Doenças Crônicas Não Transmissíveis (DCNT) no Brasil. Brasília: 2011. 160 f. [acesso em 29 março 2019]. Disponível em: http://bvsms.saude.gov.br/bvs/publicacoes/plano_acoes_enfrent_dcnt_2011.pdf

6. Portaria № 2.436 Aprova a Política Nacional de Atenção Básica, estabelecendo a revisão de diretrizes para a organização da Atenção Básica, no âmbito do Sistema Único de Saúde (SUS) 2017 set 21. Pub DO 68(69), [Set 22 2017].

7. Ministério do Desenvolvimento Social e Combate à Fome (MDS). Marco de Referência de Educação Alimentar e Nutricional para as Políticas Públicas. Brasília: 2012. 67 f. Disponível em: https://ideiasnamesa.unb.br/files/marco_EAN_visualizacao.pdf

8. Ministério da Saúde. Política Nacional de Alimentação e Nutrição (PNAN). Brasília: 2013. 86 f. [acesso em 29 março 2019]. Disponível em: http://bvsms.saude.gov.br/bvs/publicacoes/politica_nacional_alimentacao_nutricao.pdf

9. Brasil. Portaria № 2446 Redefine a Política Nacional de Promoção da Saúde 2014 nov 11. Pub DO 68(70), [Nov 13 2014].

10. Abdi J, Eftekhar H, Mahmoodi M, Shojayzadeh D, Sadeghi R, Saber M. Effect of the Intervention Based on New Communication Technologies and the Social-Cognitive Theory on the Weight Control of the Employees with Overweight and Obesity. J Res Health Sci 2015;15(4):256-61.

11. Tougas ME, Hayden JA, McGrath PJ, Huguet A, Rozario S. A Systematic Review Exploring the Social Cognitive Theory of Self-Regulation as a Framework for Chronic Health Condition Interventions. PLoS One 2015;10(8): e0134977.
12. Lima CR, Menezes IHCF, Peixoto MRG. Educação em saúde: avaliação de intervenção educativa com pacientes diabéticos, baseada na teoria social cognitiva. Ciênc Educ 2018;24(1):141-56.

13. Glanz K, Rimer BK, Viswanath KV. Health Behavior and Health Education: theory, research and practice. 4th ed. San Francisco: Jossey-Bass; 2008. 592 p.

14. Bandura A. Health promotion by social cognitive means. Health Educ Behav 2004;31:143-64.

15. Lindemann IL, Oliveira RR, Mendoza-Sassi RA. Dificuldades para alimentação saudável entre usuários da atenção básica em saúde e fatores associados. Ciênc Saúde Coletiva 2016;21(2):599-610.

16. Worsley A. Nutrition knowledge and food consumption: can nutrition knowledge change food behaviour? Asia Pacific J Clin Nutr 2002;11(Suppl):S579-85

17. Ministério da Saúde. Contribuições dos Núcleos de Apoio à Saúde da Família para a Atenção Nutricional. Brasília: 2017. 38 f. [acesso em 29 março 2019]. Disponível em: http://bvsms.saude.gov.br/ bvs/publicacoes/contribuicoes_saude_familia_atencao_nutricional.pdf

18. Fittipaldi ALM, Barros DC, Romano VF. Apoio Matricial nas ações de Alimentação e Nutrição: visão dos profissionais da Estratégia Saúde da Família. Physis 2017;27(3):793-811.

19. Cervato-Mancuso AM, Vincha KRR, Santiago DA. Educação Alimentar e Nutricional como prática de intervenção: reflexão e possibilidades de fortalecimento. Physis Rev Saúde Coletiva 2016;26(1):225-49

20. Ashman F, Sturgiss E, Haesler E. Exploring Self-Efficacy in Australian General Practitioners Managing Patient Obesity: A Qualitative Survey Study. Int J Family Med 2016;2016:8212837.

21. Mendes EV. As redes de atenção à saúde. Brasília: 2011. 549 f. [acesso em 29 março 2019]. Disponível em: http://bvsms.saude.gov.br/bvs/publicacoes/redes_de_atencao_saude.pd

22. Silva DO, Recine EGIG, Queiroz EFO. Concepções de profissionais de saúde da atenção básica sobre a alimentação saudável no Distrito Federal, Brasil. Cad Saúde Pública 2002;18(5):1367-77.

23. Bandura A. Exercise of human agency through collective efficacy. Curr Dir Psychol Sci 2000;9(3):75-8.

24. Campos GWS. Saúde pública e saúde coletiva: campo e núcleo de saberes e práticas. Ciênc Saúde Coletiva 2000;5(2):219-30.

25. Fanzo JC, Graziose MM, Kraemer K, Gillespie S, Johnston JL, Pee S, et al. Educating and Training a Workforce for Nutrition in a Post-2015 World. Adv Nutr 2015;6(6):639-47.

26. Silva JAM, Peduzzi M, Orchard C, Leonello VM. Educação interprofissional e prática colaborativa na Atenção Primária à Saúde. Rev Esc Enferm USP 2015; 49(Esp2):16-24.

27. Gucciardi E, Espin S, Morganti A, Dorado L. Exploring interprofessional collaboration during the integration of diabetes teams into primary care. BMC Fam Pract 2016:17:12 
28. Bandura A. Self-efficacy mechanism in human agency. American Psychologist 1982;37(2):122-47.

29. Einloft ABN, Cotta RMM, Araújo RMA. Promoção da alimentação saudável na infância: fragilidades no contexto da Atenção Básica. Ciênc Saúde Coletiva 2018;23(1):61-72.

30. Rigon AS, Schmidt ST, Bógus CM. Desafios da nutrição no Sistema Único de Saúde para construção da interface entre a saúde e a segurança alimentar e nutricional. Cad Saúde Pública 2016;32(3):e00164514.

\section{Colaboradores}

Reis LC conduziu a coleta de dados, analisou e interpretou os resultados e escreveu o manuscrito; Jaime PC concebeu o estudo, interpretou os resultados e revisou o manuscrito. Ambas autoras leram e aprovaram o manuscrito final. Esta pesquisa integra projeto de doutorado de LCR (Programa de Pós-graduação em Nutrição em Saúde Pública da Faculdade de Saúde Pública da Universidade de São Paulo - FSP/USP).

Conflitos de interesses: As autoras declaram não haver conflitos de interesses.

Recebido: 27 de dezembro de 2018

Revisado: 29 de março de 2019

Aceito: 29 de abril de 2019 